\title{
Nivel de comunicación escrita, deficiencias lingüísticas, y el logro de competencias en estudiantes ingresantes en una universidad pública del Cusco
}

Gabriel Suyo Cruz

gabriel.suyo@unsaac.edu.pe

ORCID ID: https://orcid.org/0000-0002-9490-403X Universidad Nacional de San Antonio Abad del Cusco

María Dolores Velasco Palacios

maria.velasco@unsaac.edu.pe

ORCID ID: https://orcid.org/0000-0001-6978-4112 Universidad Nacional de San Antonio Abad del Cusco

Josue Huamaní Cayllahua

jhuamani@unamba.edu.pe

ORCID ID: https://orcid.org/0000-0003-0404-8557

Universidad Nacional Micaela Bastidas de Apurímac

\section{RESUMEN}

En la actualidad, el entorno de educación está sufriendo grandes desafíos; es decir, la educación en el Perú, especialmente Educación Básica Regular (EBR), presenta una serie de dificultades en la redacción de los documentos administrativo y académicos. El objetivo de estudio es establecer la relación entre el nivel de comunicación escrita, las deficiencias lingüísticas, y el logro de competencias en estudiantes ingresantes en una Universidad Pública del Cusco. El enfoque de estudio es cuantitativo y aplicativo, con un diseño transversal correlacional causal; está conformada por un total de 1406 estudiantes ingresantes a la Universidad Nacional de San Antonio Abad del Cusco, eligiendo para el estudio de 142 estudiantes a través del muestreo no probabilístico por conveniencia, compilando la información mediante la escala de Likert, habiendo examinado su validez y confiabilidad. Obteniendo las conclusiones fundamentales que, si existe una asociación significativa entre el nivel de comunicación escrita, las deficiencias lingüísticas, y el logro de competencias en estudiantes ingresantes en una Universidad Pública del Cusco. Así como, se observa con el contraste de la prueba de hipótesis cuyo "P-value" $=0,000$ es menor que el valor de significancia 0,05. Evidencia mediante la prueba estadístico de Rho Spearman se deniega la hipótesis nula, y admitimos la hipótesis alternativa.

Palabras clave: nivel de comunicación escrita; deficiencias lingüísticas; logro de competencias. 


\title{
Level of written communication, linguistic deficiencies, and the achievement of competences in incoming students in a public university of Cusco
}

\begin{abstract}
Currently, the education environment is experiencing great challenges; In other words, education in Peru, especially Regular Basic Education (EBR), presents a series of difficulties in the writing of administrative and academic documents. The objective of the study is to establish the relationship between the level of written communication, the linguistic deficiencies, and the achievement of competences in students entering a Public University of Cusco. The study approach is quantitative and applicative, with a causal correlational cross-sectional design; It is made up of a total of 1406 students entering the National University of San Antonio Abad del Cusco, choosing for the study of 142 students through non-probability sampling for convenience, compiling the information using the Likert scale, having examined its validity and reliability. Obtaining the fundamental conclusions that if there is a significant association between the level of written communication, linguistic deficiencies, and the achievement of competencies in students entering a Public University of Cusco. As well as, it is observed with the contrast of the hypothesis test whose "P-value" $=0.000$ is less than the significance value 0.05 . Evidence using the Rho Spearman statistical test denies the null hypothesis, and we admit the alternative hypothesis.
\end{abstract}

Keywords: level of written communication; linguistic deficiencies; achievement of competences.

Artículo recibido: 10. Junio. 2021 Aceptado para publicación: 16. Julio. 2021 Correspondencia: gabriel.suyo@unsaac.edu.pe Conflictos de Interés: Ninguna que declarar 


\section{INTRODUCCIÓN}

La comunidad de la cognición y de la comunicación ha innovado las formas del empleo, los procesos de organización social, económica, cultural, filosófica, ideológica y educativa en la humanidad desde hace más de tres décadas. Lo que ha conllevado a enfrentar nuevos desafíos, nuevos conceptos de aprendizaje y el dominio de competencias, tanto a nivel individual, e interpersonal, social y laboral, bajo un enfoque de enseñanza a largo y ancho de la vida.

La educación superior como las universidades públicas buscan actualmente que la sociedad mejore en la comunicación, la responsabilidad de crear conocimientos en estas aulas universitarias y todo ello nos lleva a una problemática general (Arciniegas Lagos y López Jiménez, 2012). Ayudan brindar a que los estudiantes de Estudios Generales desarrollen conocimientos, así inician el aprendizaje diario; con ello se logra mejorar las competencias y esto refleja directamente en su producción de textos. En efecto, la disciplina lingüística es fundamental para los alumnos de pregrado, en las escuelas profesionales, deben tener conocimientos del tiempo y sobre la capacidad del facilitador para apoyar en el proceso de su redacción del discurso.

El proceso de la comunicación escrita que se tiene con los estudiantes es un factor preponderante donde la escritura explícita de la triangulación de la oralidad, en donde estos elementos son indispensables al momento de realizar intercambio de información y experiencia. El emisor es la persona que inicia la comunicación, el oyente recepciona el informe del hablante y el contenido se encuentra en el mensaje. Y todo esto, se debe manejar un código para que existe una comunicación efectiva.

La deficiencia semántica se asocia con el plano del contenido del signo lingüístico con sentido e interpretación de las palabras; así mismo, en las expresiones debe tener sentido y autonomía sintáctica para poder asimilar en la comunicación escrita y oral. El hablante y el oyente deben manejar los términos técnicos relacionados a la especialidad para que exista una comunicación eficiente, esto implica que el estudiante debe practicar la lectura para familiarizarse con las palabras, la cual lleva un sentido pragmático social.

A nivel de estudiantes universitarios de nivel superior no practican con seriedad la redacción de los documentos académicos, administrativos, literarios y científicos. Esto se observa en la producción de sus textos que no realizan como debe ser, donde prima el desinterés, apatía, indiferencia al momento de producir los diferentes textos. Así mismo, 
las evidencias de aprendizajes tienen deficiencias: Semánticas, sintácticas, gramaticales, morfológicas, fonológicas y pragmáticas. Por ello, se propone trabajar con los estudiantes de nivel superior, para mejorar sus competencias genéricas de aprendizaje que servirán de ayuda en formación académica y científica. Todo esto, se resume en el logro de competencias de los estudiantes ingresantes a una Universidad Pública.

Por ende, los estudiantes de todas las escuelas profesionales deben tener manejo y conocimiento de la expresión oral y escrita, para poner en práctica la producción de los diferentes textos y esto servirá para mejorar en su formación profesional académica, científica y tecnológica.

En la producción de textos participa un papel fundamental y sustancial el desarrollo de enseñanza aprendizaje, primordialmente la interpretación trasparente que no se procesa en su conjunto, menos la acumulación y simplicidad de ideas para formar en un argumento del contexto.

En realidad, en los estudiantes de Estudios Generales de la Universidad Nacional de San Antonio Abad del Cusco (UNSAAC), se observa diferentes realidades problemáticas, especialmente en la comunicación escrita. Así mismo, en la expresión oral tienen dificultades de enfrentarse al escenario y sustentar sus exposiciones de trabajos encargados. Del mismo modo, los estudiantes tienen déficit en comunicación porque no utilizan correctamente el código lingüístico, en donde se presentan incoherencias en sus expresiones y al mismo tiempo reflejan en sus producciones escritas. Así, se nota que una frase ocupa un párrafo y el estudiante ya no tiene coherencia en el significado de las palabras que expresa. Además, construyen oraciones extensas sin pausas sin uso de las reglas ortográficas gramaticales adecuados, los cuales influye directamente en sus expresiones, asimismo redactar textos cortos y extensos. Así, en muchas circunstancias se observa carencia de creatividad para mejorar y argumentar sus pensamientos, ideas, que utilizan para producir textos. Finalmente, requiere estos estudiantes enriquecer en sus léxicos para mejorar en su comunicación oral y escrita. En atención a lo cual, la investigación se planteó como pregunta: ¿Qué relación existe entre el nivel comunicación escrita, las deficiencias lingüísticas, y el logro de competencias en estudiantes ingresantes en una Universidad Pública del Cusco? 


\subsection{Comunicación escrita}

Fonseca Yerena, Correa Pérez, Pineda Ramírez, y Lemus Hernández (2011) manifestaron que la comunicación es la interacción social que se da entre los interlocutores. Gutiérrez Castillo, López Heredia, Salazar González, y Ibarra Ramírez (2012) manifestaron que es la interacción de la información hacia uno mismo. Con relación, Rojas Saldaña (2013) indicó que la comunicación es el intercambio del proceso comunicativo, que realiza utilizando signos lingüísticos y sirven, para informar y recordar significados. Es decir, comunicar es dar a conocer todo a aquello que evocamos de experiencias haciendo uso de la comunicación lingüística y no lingüística.

Actualmente, en las universidades se ha quedado desfasado la enseñanza en relación a la lengua española; es decir, la enseñanza de Educación Básica Regular con la Universidad no tiene una articulación adecuada; por esta razón, se tiene estos desniveles o desfases y no hay una concreción real. Por otro lado, se tiene que poner en práctica los conocimientos teóricos científicos que estén relacionados con las ciencias lingüísticas, como: Sociolingüística, psicolingüística, pragmática, lingüística textual, análisis del discurso y la didáctica.

Pinales Rodríguez y Lagunas Beltrán (1998, p. 21) afirmaron que la lengua es habilidad ejecutable en los recursos de los hablantes de la lengua, que tiene a su orden; así mismo, es una actitud que tiene sentido, coherencia, disponibilidad y posibilidad de ejecución, para explicar lo que quiere y se siente para informar inclusive una visión individual y personal.

La lengua es un sistema de signos lingüísticos; es decir, es la aptitud que tiene el individuo para explicar a través de un sistema de comunicación integrado, ya sean por signos orales o escritas que forman parte de un contexto lingüístico definido. Entonces la lengua es una estructura convencional de signos realizados por la sociedad para realizar sus comunicaciones de manera eficiente y efectiva.

Toda redacción tiene una estructura que compone tres elementos relevantes como; introducción, desarrollo y cierre:

a) Introducción: Es la iniciación de la producción de texto, en donde plasma el resumen situacional, lugar, tiempo y la secuencia histórica.

b) Desarrollo: Donde se explica todo el desarrollo del contenido de la investigación. . 
c) Cierre: Se concluye con la solución de la secuencia del contenido de la investigación de forma resumida y sintética.

Cortés Moreno (2000, p. 34) presentó que la escritura es la base fundamental de toda persona que requiere construir palabras, frases, oraciones y textos. En este entender, la escritura ayuda a desarrollar habilidades y destrezas en los individuos y con la pragmática ayuda mejorar el ejercicio en el desarrollo cognoscitivo, lingüístico, sentimental y social. En la actualidad evidenciamos una innovación rápida y relevante en los instrumentos que suministran conocimiento y la comunicación. Apreciamos las innovaciones que se dan en la comunidad para la utilización de nuevas formas de comunicarse a través de nuevos formatos virtuales y plataformas digitales, donde la comunicación es mediata y establece con la accesibilidad disponible y libre. La comunicación escrita está implícita en las disciplinas lingüísticas (semántica, semiótica, fonética, fonología, sintaxis, ortografía y gramática), las cuales regulan las normas propias de las variaciones lingüísticas, sino que dicha habilidad integra la necesidad comunicativa intrínseca de la persona (Estévez Fuertes et al., 2011).

\section{Fines de la comunicación escrita}

Para considerar en cuanto a los fines de la comunicación escrita, es necesario aceptar que al lenguaje oral como medio de comunicación expresiva, los fines principales son dos: Reflexionar y comunicarse; es decir, expresar el texto con nuestras propias palabras y/o experiencias, para luego transformar en un nuevo conocimiento, pero respetando el significado original, con la finalidad de llegar a un entendimiento mejor.

De tal forma, la finalidad de la comunicación escrita es concretizar la información del lector en una forma adecuada para expresar pensamientos similares de lo que trasmite el autor del texto escrito. Para que logre su objetivo de la comunicación escrita debe considerarlo siguiente:

- Escribir para el lector.

- Establecer las expresiones y presentar a los lectores sin que expresen malas apreciaciones.

- Utilizar palabras legibles, perceptibles y expresiones que tengan sentido completo.

- Difundir y examinar detalladamente el primer escrito.

Según Estévez Fuertes et al. (2011) consideraron las siguientes dimensiones de comunicación escrita se basa en redacción científica, literaria y administrativa. Es decir, 
La escritura es una forma de diálogo que permite al individuo comunicarse a partir de un documento; dicho de otra manera, es una manera de intercambiar información entre dos personas. En este el hablante produce diferentes textos continuos (artículos académicos, ensayos, novelas, cuentos literarios, trabajos monográficos, interpretación de textos, entre otros), con lo que busca informar su mensaje a los destinatarios o receptores.

\subsection{Deficiencias lingüísticas}

Alonso-Cortés Manteca (2002, p. 11) expresó que "es el estudio científico de los idiomas como expresión internacional de la palabra". Por su lado, Mounin y Ter-Sakarian (1979, p. 115) definen que la ciencia del leguaje es el estudio de los propósitos y características de la composición en el orden y desarrollo en el contexto y el espacio de las lenguas naturales humanas. De la misma manera, los intelectuales citados tienen similitud en concretizar las características científicas de la lingüística; tomando en cuenta la formación pedagógica de los fenómenos que cooperan en el desarrollo de las lenguas naturales y el manejo del conocimiento de los interlocutores, con variaciones que se dan en el tiempo y espacio, finalmente, sin variación es estática. En resumen, la ciencia de lingüística expresa todas las manifestaciones de lenguaje; es decir, el conocimiento de la investigación científica del tema.

Integrando las palabras deficiencia y lingüística, significa que es la carencia o falta de propiedades de la lengua natural; así mismo, en el manejo del conocimiento y la pragmática de los interlocutores. A este trabajo de investigación se incluye las disciplinas lingüísticas: Ortografía, pragmática, semántica, morfología y sintaxis. Estos recursos lingüísticos es base fundamental para el dominio lingüístico. Así como expresa, Martínez Martínez (2002) es una técnica y estratégica que no funcionan adecuadamente en la utilización de la semántica y la pragmática; por tanto, admiten los requisitos pertinentes de modo importante de individuo con terceros.

Primeramente, hay que distinguir que son las debilidades y dificultades particulares en el contexto del habla y del lenguaje; es decir, habla es expresión individual de la persona y el lenguaje es la capacidad para expresar pensamientos e ideas. En este entender, se agrega la diferencia entre la comunicación, lenguaje y habla. O sea, la comunicación ocupa de la interacción entre dos o más personas, en la mayoría de las ocasiones o circunstancias utilizan el lenguaje lingüístico y no lingüístico. En seguida el lenguaje es la capacidad de hablar ideas, sentimientos, tendencias, a través del uso de signos verbales, 
y no verbales, mediante los cuales se expresan experiencias. Finalmente, el habla se refiere a la expresión particular del individuo de esta forma tiene incorporado los mecanismos fisiológicos y neurológicos. Si bien, cada una de estas palabras presentadas demuestran que son diferentes en el contexto significado, de esta forma evocar que cada uno tiene una interrelación constante entre estos términos. (Godoy Lenz y Zilliani Illanes, 2007)

Según Martínez Solís (2002, p. 46) manifestó que las dimensiones de la deficiencias lingüísticas considera déficit semántico, déficit sintáctico, déficit morfológico, y déficit ortográfico. Es decir, la construcción de textos sigue una estructura dependiendo del tipo de este, para luego explicar a los grupos el significado pertinente. De esta manera, se observa que hay carencia en el manejo de las normas ortográficas gramaticales, para la elaboración de diferentes documentos.

\subsection{Competencias}

El significado de aptitud tiene una estrecha relación con la habilidad para manejar diversas situaciones complejas, y esto implica que la "competencia" es fundamental que se aplique en los niveles de sapiencias y la experiencia de las destrezas para exponer y así, llevar a la comunicación de una manera efectiva.

Snyder y Ebeling (1992) explicaron en relación a la competencia desde la perspectiva funcional, sin embargo, utilizan "competencies" en plural. Otros escritores frecuentemente utilizan el término "competency" cuando habla sobre la competencia ocupacional o se emplean como sinónimo. Por lo tanto, Dale (1992) diferencia las habilidades ocupacionales de las particularidades psicosociales, sin embargo utilizan competence y competency para ejecutar la descripción en la discusión de su documento para examinar sus habilidades y destrezas. Hartle (1995) manifestó que la competency como "la particularidad que una persona tiene manejo en la producción de su trabajo", incorpora las "competencias" observables (conocimientos y habilidades) como "resúmenes de competencias" (rasgos y motivos).

\section{Dimensiones de competencias}

García Cabrero, Loredo Enríquez, Luna Serrano, y Rueda Beltrán (2008) y el Bravo Salinas (2007), y Beneitone et al. (2007) en su publicación de su libro de "Reflexiones y perspectivas de la Educación Superior en América Latina”, dan a conocer sobre la clasificación de las competencias: 
Competencias genéricas: ("generales" o "transversales"), que son los más usuales en toda las profesiones y ocupaciones; que integran el conjunto de conocimientos generales que tienen que conocer todo egresado de la universidad (García Cabrero et al., 2008). Por otra parte, en su formación los estudiantes tienen que conocer los diferentes tópicos relacionados con la estructuración gramatical. Estos temas se relacionan entre sí, para tener una comunicación oral y escrita eficiente entre los interlocutores, y forma parte como en su formación académica formando verdaderamente para enfrentarse y servir a la sociedad, de acuerdo a su especialidad (Beneitone et al., 2007, p. 15).

Competencias específicas: Este tipo de competencias son conocidas como partículas o especialización en la formación de cada individuo (García Cabrero et al., 2008, p. 102). Distinguir la formación en una especialidad frente a otra; además, existen especializaciones con manejo de término de especialidad, las cuales inciden en su capacitación personal y académica (Beneitone et al., 2007, p. 16).

Por lo referido, se formuló como problema general: ¿De qué manera el nivel comunicación escrita, las deficiencias linguísticas, se relaciona con el logro de competencias en estudiantes ingresantes en una Universidad Pública del Cusco?; Como objetivo general, se propuso: Establecer la relación existente entre el nivel de comunicación escrita, las deficiencias lingüísticas, y el logro de competencias en estudiantes ingresantes en una Universidad Pública del Cusco. Y como objetivos específicos: Identifica el nivel de comunicación escrita en los estudiantes ingresantes en una Universidad Pública del Cusco. Identificar el nivel de las deficiencias lingüísticas en los estudiantes ingresantes en una Universidad Pública del Cusco. Describir el logro de competencias en los estudiantes ingresantes en una Universidad Pública del Cusco.

\section{MATERIALES Y MÉTODOS}

La metodología es aplicativa con enfoque de investigación cuantitativo y el estudio es descriptivo - correlacional. Primero describir las variables tal como se encuentra en la observación; segundo el nivel de relación de las variables de estudio. El diseño de investigación es no experimental porque no se alteraron las variables de estudio, se detallan y tal cual se presenta en el estudio de campo (Toro Jaramillo y Parra Ramírez, 2006). El estudio de la población está conformado por los alumnos ingresantes de la Universidad Nacional de San Antonio Abad del Cusco (UNSAAC), que suman un total de 1406 estudiantes ingresantes por diferentes modalidades a la UNSAAC del año 2019 
(Unidad de Centro de Cómputos de la UNSAAC). La muestra es de tipo no probabilístico por el método de muestreo por conveniencia a los estudiantes de la Escuela Profesional de Educación - UNSAAC., y está conformada por 150 estudiantes ingresantes.

La técnica que se empleó es la encuesta, que han sido elaborados de acuerdo a las dimensiones y los indicadores de las variables; como instrumento empleado es el cuestionario de preguntas, el cual estuvo orientado a medir cada uno de las variables, poseyendo como colaboradores a los alumnos ingresantes a la Escuela Profesional de Educación - UNSAAC. Una vez conseguida los datos de las encuestas, se presenta la validación de los contenidos con juicio de expertos, las cuales son personas conocedoras del tema. En seguida, se realizó la confiabilidad con el coeficiente alfa Cronbach para medir la interna de las preguntas, estableciendo la media de las correlaciones entre los ítems (Cronbach, 1951, p. 299). Para el procesamiento de las tablas y figuras se utilizó, programa Microsoft Excel y el desarrollo de estadística descriptiva e inferencia se empleó el software estadístico SPSS.

Para la descripción de las variables, se empleó la estadística descriptiva y para la interpretación de los resultados se realizó la baremización para cada uno de las variables; del mismo modo, se efectuó para las dimensiones el baremo de interpretar e identificación de las categorías, es por ello la escala de evaluación es ordinal. La identificación de nivel de comunicación toma como atributos de: Muy bajo, bajo, moderado y alto. Mientras, para las deficiencias lingüísticas considera: Bajo, medio, alto, muy alto. Finalmente, para la variable de logro de competencias toma los atributos de: Deficiente, regular, bueno, y muy bueno.

La presentación de análisis inferencial inicia con la prueba de normalidad, para observar el grado de distribución normal de los datos. Así mismo, se usó la prueba de Test de Kolmogorov-Smirnov, teniendo en cuenta el resultado de la normalidad, que los datos difieren de la distribución normal (no paramétrico). Por consiguiente, se elegio emplear la prueba de hipótesis correlacional de Rho de Spearman.

\section{RESULTADOS Y DISCUSIÓN}

\subsection{Resultados}

El trabajo de investigación, presenta los resultados que fueron interpretados en primera instancia el nivel descriptivo y en seguida el nivel inferencial. En primer lugar, se han 
utilizado la estadística descriptiva - relativa, sosteniendo como fundamento las frecuencias absolutas y las frecuencias relativas de los investigados.

Tabla 1. Resultados de la distribución de la variable de nivel de comunicación escrita

\begin{tabular}{|c|c|c|c|c|c|c|c|c|c|c|}
\hline \multirow{3}{*}{ Dimensiones } & \multicolumn{8}{|c|}{ Variable de nivel de comunicación escrita } & \multicolumn{2}{|c|}{ Total } \\
\hline & & uy bajo & & Bajo & Mo & derado & & Alto & & \\
\hline & $f$ & $\%$ & $f$ & $\%$ & $f$ & $\%$ & $f$ & $\%$ & J & $\%$ \\
\hline $\begin{array}{l}\text { Redacción científica } \\
\text { tecnológica }\end{array}$ & 28 & $19,72 \%$ & 62 & $43,66 \%$ & 40 & $28,17 \%$ & 12 & $8,45 \%$ & 142 & 100 \\
\hline Redacción literaria & 33 & $23,24 \%$ & 61 & $42,96 \%$ & 36 & $25,35 \%$ & 12 & $8,45 \%$ & 142 & 100 \\
\hline Redacción administrativa & 34 & $23,94 \%$ & 64 & $45,07 \%$ & 28 & $19,72 \%$ & 16 & $11,27 \%$ & 142 & 100 \\
\hline
\end{tabular}

Nota: Datos procesados en el programa estadístico SPSS.

En la Tabla 1, se presentan los fundamentos consolidados de los porcentajes de la dimensión de redacción científica tecnológica; el 43,66\% de los encuestados señalaron que nivel es bajo. Así mismo, el 28,17\% manifestaron que está en un nivel moderado. Por otro lado, el 19,72\% consideran que están en un nivel bajo. Por lo tanto, que la mayor parte de los encuestados expresaron que la redacción científica tecnológica está en un nivel bajo y muy bajo en cuanto en sus redacciones de textos que realizan en sus diferentes materias de estudio; así mismo, los estudiantes comprenden que en su proceso de aprendizaje es de vital importancia para las actividades y necesitan comunicarse con coherencia, cohesión, claridad y formalidad que sus expresiones sean correctas.

Los resultados consolidados de la dimensión de redacción literaria; el 42,96\% de los estudiantes indicaron que están en un nivel bajo. Del mismo modo, el 25,35\% revelaron que está en un nivel moderado. Por último, el 23,24\% indicaron que están en un nivel muy bajo. Finalmente, la gran parte de los encuestados expresaron que si están en un nivel bajo y muy bajo en cuanto a la redacción literaria; es decir, los estudiantes no tienen una cultura de lectura de algunas obras para una reflexión de los hechos y sucesos que se presentaron en nuestro país, desde este enfoque se valora la importancia de la redacción de textos literarios y la interpretación que deben tener una coherencia con el contexto.

En referente, a los resultados consolidados de la dimensión de redacción administrativa; el 45,07\% de los estudiantes manifestaron que están en un nivel bajo. En seguida, el 23,94\% declararon que está en un nivel muy bajo. Así mismo, el 19,72\% expresaron que están en un nivel moderado. En conclusión, la gran parte de los encuestados expresaron que si están en un nivel bajo y muy bajo en la redacción administrativa literaria; es decir, 
los estudiantes ingresantes no conocen todavía los documentos administrativos que establecen una redacción adecuada para comunicarse en forma clara y preciso.

Tabla 2. Resultados de la distribución de la variable de las deficiencias lingüísticas

\begin{tabular}{|c|c|c|c|c|c|c|c|c|c|c|}
\hline \multirow{3}{*}{ Dimensiones } & \multicolumn{8}{|c|}{ Variable de las deficiencias lingüísticas } & \multicolumn{2}{|c|}{ Total } \\
\hline & \multicolumn{2}{|c|}{ Bajo } & \multicolumn{2}{|c|}{ Medio } & \multicolumn{2}{|c|}{ Alto } & \multicolumn{2}{|c|}{ Muy alto } & \multirow[b]{2}{*}{$f$} & \multirow[t]{2}{*}{$\%$} \\
\hline & $f$ & $\%$ & $f$ & $\%$ & $f$ & $\%$ & $f$ & $\%$ & & \\
\hline Déficit semántico & 10 & $7,04 \%$ & 26 & $18,31 \%$ & 65 & $45,77 \%$ & 41 & $28,87 \%$ & 142 & 100 \\
\hline Déficit sintáctico & 10 & $7,04 \%$ & 34 & $23,94 \%$ & 58 & $40,85 \%$ & 40 & $28,17 \%$ & 142 & 100 \\
\hline Déficit morfológico & 8 & $5,63 \%$ & 37 & $26,06 \%$ & 52 & $36,62 \%$ & 45 & $31,69 \%$ & 142 & 100 \\
\hline Déficit ortográfico & 8 & $5,63 \%$ & 37 & $26,06 \%$ & 56 & $39,44 \%$ & 41 & $28,87 \%$ & 142 & 100 \\
\hline
\end{tabular}

Nota: Datos procesados en el programa estadístico SPSS.

En la Tabla 2, se presenta los resultados consolidados de los porcentajes de la dimensión del déficit semántico; el 45,77\% de los estudiantes encuestados manifestaron que está en un nivel alto. Así mismo, el 28,87\% expresaron que está en un nivel muy alto. Por otro lado, el 18,31\% indicaron que están en un nivel medio. Para terminar, la gran parte de los encuestados indicaron sobre las deficiencias en déficit semántico están en un nivel alto y parte está en muy alto en cuanto en sus estructuraciones gramaticales que realizan en sus trabajos monográficos y se observa que estos tienen dificultades en las interpretaciones de los significados de las palabras, el cual está relacionado con el nivel de comunicación escrita.

En referente a los resultados consolidados de la dimensión de déficit sintáctico; el 40,85\% de los estudiantes encuestados indicaron que está en un nivel alto. Del mismo modo, el $28,17 \%$ expresaron que está en un nivel muy alto. En seguida, el 23,94\% consideraron que están en un nivel medio. En atención a lo cual, la gran parte de los investigados reconocieron que si están en un nivel medio y bajo en las deficiencias lingüísticas fundamentalmente en déficit sintáctico; es decir, los estudiantes ingresantes a la universidad tienen dificultades en las construcciones de sus textos y así mismo, en la concordancia de las oraciones que corresponda a una coherencia, cohesión, adecuación en la redacción de oraciones, frases y textos.

Los resultados consolidados de la dimensión de déficit morfológico; el 36,62\% de los estudiantes encuestados manifestaron está en un nivel alto. En seguida, el 31,69\% encuestados declararon que está en un nivel muy alto. En seguida, el 26,06\% expresaron que están en un nivel medio. En conclusión, la gran parte de los encuestados expresaron que si están en un nivel alto y al mismo tiempo consideran muy alto en déficit 
morfológico; es decir, los estudiantes ingresantes dificultan en cuanto a reconocer las diferentes formas de las palabras en su estructuración gramatical, las cuales influye en las redacciones de los diferentes textos académicos, científicos y administrativos.

Los resultados consolidados de la dimensión de déficit ortográfico; el 39,44\% de los estudiantes encuestados indicaron que está en un nivel alto. Del mismo modo, el 28,87\% dos expresaron que está en un nivel muy alto. En seguida, el 26,06\% consideran que están en un nivel medio. En conclusión, la gran parte de los encuestados explicaron que si están en un nivel alto y muy alto en las deficiencias lingüísticas en especial en déficit ortográfico; es decir, los estudiantes ingresantes a la universidad tienen problemas en la conjugación gramatical o con la forma de escribir las palabras, tomando en cuenta las normas ortográficas y gramaticales.

Tabla 3. Resultados de la distribución de la variable de logro de competencias

\begin{tabular}{|c|c|c|c|c|c|c|c|c|c|c|}
\hline \multirow{3}{*}{ Dimensiones } & \multicolumn{8}{|c|}{ Variable logro de competencias } & \multirow{3}{*}{\multicolumn{2}{|c|}{$\begin{array}{l}\text { Total } \\
f \quad \%\end{array}$}} \\
\hline & \multicolumn{2}{|c|}{ Deficiente } & \multicolumn{2}{|c|}{ Regular } & \multicolumn{2}{|c|}{ Bueno } & \multicolumn{2}{|c|}{ Muy bueno } & & \\
\hline & $f$ & $\%$ & $f$ & $\%$ & $f$ & $\%$ & $f$ & $\%$ & & \\
\hline Logro & 18 & $12,68 \%$ & 68 & $47,89 \%$ & 44 & $30,99 \%$ & 12 & $8,45 \%$ & 142 & 100 \\
\hline
\end{tabular}

Nota: Datos procesados en el programa estadístico SPSS.

En la Tabla 3, se muestra resultados consolidados de los porcentajes de la dimensión logro de competencias; el 47,89\% de los estudiantes encuestados manifestaron que está en un nivel de regular. Así mismo, el 30,99\% expresaron que está en un nivel bueno. Por otro lado, el 12,68\% manifestaron que están en un nivel deficiente. Para terminar, nos presenta que la gran parte de los encuestados nos indicaron que el logro de competencias está en un nivel regular y bueno en cuanto en su aplicación del curso de redacción de textos buscamos mejorar las competencia genéricas y específicas de los estudiantes ingresantes a la UNSAAC.

\section{Prueba de normalidad}

Tabla 4. Test de prueba de Kolmogorov-Smirnov para una muestra

\begin{tabular}{llrrr} 
& & $\begin{array}{c}\text { Nivel de } \\
\text { comunicación escrita }\end{array}$ & $\begin{array}{c}\text { Deficiencias } \\
\text { lingüísticas }\end{array}$ & $\begin{array}{c}\text { Logro de } \\
\text { competencias }\end{array}$ \\
\hline $\mathrm{N}$ & 142 & 142 & 142 \\
\hline \multirow{2}{*}{$\begin{array}{l}\text { Parámetros } \\
\text { normales }\end{array}$} & Media & 2,52571 & 2,32831 & 2,94443 \\
& Desviación & 1,09289 & 1,091776 & 1,18190 \\
\hline \multirow{2}{*}{$\begin{array}{l}\text { Máximas diferencias } \\
\text { extremas }\end{array}$} & Abtándar &, 242 &, 153 &, 187 \\
& Absoluto &, 242 &, 138 &, 144 \\
\hline Estadístico de prueba & Positivo &,- 153 &,- 176 &,- 187 \\
Sig. asintótica(bilateral) & &, 271 &, 247 &, 255 \\
\hline
\end{tabular}

Nota: Datos procesados en el programa estadístico SPSS. 
Los resultados que demuestran que la mayoría de las variables tienen puntuaciones y no proceden de una distribución normal, siendo las variables cuantitativas continua. Por lo tanto, elegimos emplear la comprobación de hipótesis correlacional estadístico no paramétrico Rho de Spearman (por tener variables categóricas y numéricas), puesto que no cumplen el supuesto de normalidad.

Tabla 5. Correlación de nivel de comunicación escrita, deficiencias lingüísticas, y logro de competencias en los estudiantes ingresantes

Correlaciones

\begin{tabular}{|c|c|c|c|c|c|}
\hline & & & $\begin{array}{c}\text { Nivel de } \\
\text { comunicación } \\
\text { escrita }\end{array}$ & $\begin{array}{l}\text { Deficiencias } \\
\text { lingüísticas }\end{array}$ & $\begin{array}{c}\text { Logro de } \\
\text { competencias }\end{array}$ \\
\hline \multirow{9}{*}{$\begin{array}{l}\text { Rho de } \\
\text { Spearman }\end{array}$} & \multirow{3}{*}{$\begin{array}{l}\text { Nivel de } \\
\text { comunicación } \\
\text { escrita }\end{array}$} & Coeficiente de correlación & 1 &, $638 * *$ &, $726 * *$ \\
\hline & & Sig. (bilateral) & &, 000 & 000 \\
\hline & & $\mathrm{N}$ & 142 & 142 & 142 \\
\hline & \multirow{3}{*}{$\begin{array}{l}\text { Deficiencias } \\
\text { lingüísticas }\end{array}$} & Coeficiente de correlación & ,638** & 1 &, $651 * *$ \\
\hline & & Sig. (bilateral) &, 000 & & 000 \\
\hline & & $\mathrm{N}$ & 142 & 142 & 142 \\
\hline & \multirow{3}{*}{$\begin{array}{l}\text { Logro de } \\
\text { competencias }\end{array}$} & Coeficiente de correlación &, $726 * *$ & $651 * *$ & 1 \\
\hline & & Sig. (bilateral) &, 000 & 000 & \\
\hline & & $\mathrm{N}$ & 142 & 142 & 142 \\
\hline
\end{tabular}

**. La correlación es significativa al nivel 0,01 (bilateral).

Al emplear la prueba estadístico de correlación Rho de Spearman, se demuestra una influencia positiva significativa moderada $(r=0,638 * *$ y $\mathrm{P}=0,000)$ y directamente proporcional; es decir, existe una asociación entre el nivel de comunicación escrita y las deficiencias lingüísticas en alumnos ingresantes en una Universidad Pública del Cusco. Así mismo, se presenta una relación positiva significativa moderada buena $\left(r=0,726^{* *}\right.$ y $\mathrm{P}=0,000)$ y directamente proporcional; es decir, existe una relación directa positiva entre el nivel de comunicación escrita y el logro de competencias en estudiantes ingresantes en una Universidad Pública del Cusco. Finalmente, se presenta una influencia positiva significativa moderada $(\mathrm{r}=0,651 * *$ y $\mathrm{P}=0,000)$ y directamente proporcional; es decir, existe una relación directa positiva de las deficiencias lingüísticas y logro de competencias en estudiantes ingresantes en una Universidad Pública del Cusco, debido a que el $\mathrm{p}<0,01$ se deniega la hipótesis nula, y se admite la hipótesis alternativa.

El nivel de correlación es positivo considerablemente, de acuerdo a la tabla de interpretación de valor "Rho de Spearman", porque el factor de asociación es 0,638; 0,726 y 0,651 . Mientras exista un nivel alto de nivel de comunicación escrita y deficiencias lingüísticas habrá un buen nivel de logro de competencias en estudiantes ingresantes en una Universidad Pública del Cusco. Se concluye que las variables de nivel de 
comunicación escrita, deficiencias lingüísticas se relacionan significativamente con el logro de competencias en estudiantes ingresantes en una Universidad Pública del Cusco.

\subsection{Discusión}

El trabajo de investigación efectuó una comprobación de la validez y confiabilidad de los instrumentos del trabajo obteniendo dentro de los rangos aceptados y favorables para su aplicación de dichos instrumentos. Así mismo, validaron los expertos con su aporte de aplicabilidad de los instrumentos, lo cual generan una confianza en el empleo del documento.

A nivel descriptivo, ha permitido que los estudiantes encuestados expresaron un nivel medio en la medición de las tres variables de estudio. Los resultados que se obtiene presentan diferentes falencias en la comunicación escrita, deficiencias lingüísticas y logro de competencias; es decir, en sus producciones de párrafos deben tener claridad y precisión en la redacción.

Los estudiantes ingresantes a diferentes Escuelas Profesionales muestreados, que en su gran mayoría son procedentes de las instituciones educativas estatales y de las provincias respectivas. Lo cual se observa que el Diseño Curricular Nacional del Perú está estructurado de manera genérica y los docentes especialidad diversifican y contextualizan a su contexto articulando a la realidad de su provincia. Existiendo, desarticulación y desfase con la universidad y las instituciones educativas en los contenidos de las rutas de aprendizaje. Además, los docentes de las diferentes especialidades no trabajan con los estándares establecidos en las normas de educación, si no que simplifican los tópicos y este mismo influye en su preparación didáctica de los estudiantes de las instituciones educativas, cuando ingresan a las universidades.

Se observa que los estudiantes en la asignatura de Redacción de Textos, muestran estos casos en los ingresantes que tienen déficit en su formación académica, en la praxis no se visualiza su aprendizaje de calidad. Esto incide directamente en su utilización de las palabras, producción de textos, manejo de un lenguaje familiar. El resultado que se tiene es falta de la praxis de la lectura en todos sus niveles. El principal factor de estos estudiantes es que tengan una actitud positiva de mejorar las deficiencias y proyectarse en su formación profesional para la vida, con actitudes, habilidades y destrizas que fortalezca la calidad educativa. 
A nivel inferencial, analizamos el propósito fundamental del trabajo de estudio y se ha confirmado con la relación existente entre el nivel de comunicación escrita, las deficiencias lingüísticas, y logro de competencias en estudiantes ingresantes; tal como se evidencia con la aplicación de la prueba estadístico de Rho de Spearman en la Tabla 5, mención una relación positiva con $\mathrm{r}=0,638^{* *}$, lo que expresa que existe una asociación significativa; por otro lado, el $\rho$-valores menor del $5 \%$ y en consecuencia existe una asociación entre el nivel de comunicación escrita y las deficiencias lingüísticas en alumnos ingresantes en una Universidad Pública del Cusco. Así mismo, se evidencia una asociación positiva significativa moderada buena $\left(r=0,726^{*} *\right.$ y $\left.\mathrm{P}=0,000\right)$; es decir, existe una asociación clara positiva moderada entre el nivel de comunicación escrita y el logro de competencias en alumnos ingresantes en una Universidad Pública del Cusco. Finalmente, evidencia una influencia positiva significativa moderada buena $(r=0,651 * *$ y $\mathrm{P}=0,000)$ y directamente proporcional; es decir, existe una relación directa positiva de las deficiencias lingüísticas y logro de competencias en estudiantes ingresantes en una Universidad Pública del Cusco. En conclusión, se deniega la hipótesis nula (Ho), y se admite la hipótesis alterna (Ha), por este motivo que, si existe una relación significativa entre el nivel de comunicación escrita, las deficiencias lingüísticas, y el logro de competencias en estudiantes ingresantes en una Universidad Pública del Cusco.

Los resultados concuerdan con otras investigaciones de Gavidia Anticona (2018), donde se concluye y confirma que si existe una asociación positiva y directa entre las deficiencias lingüísticas y con la comunicación lingüística; además, expresaron que mayor déficit es en la comunicación escrita especialmente en déficit semántico en un nivel regular.

Así mismo, se ha conseguido cotejar con la investigación Bardales Balarezo (2017) los resultados fundamentales y el empleo de las reglas en el procedimientos de contrataciones existe deficiencias en los reglamentos institucionales. Cabe precisar que los resultados muestran una coincidencia en gran medida con otros estudios desarrollados indican que, a mayor presencia e intensidad que el nivel comunicación escrita está relacionado con las deficiencias lingüísticas; es decir, las variables de estudio de investigación tienen una relación entre sí.

Por otro lado, existe una semejanza con la investigación de Rojas Saldaña (2013) quien presentó una tesis doctoral, con el tema: "Deficiencias lingüísticas en relación a la 
comunicación escrita en estudiantes del ISTP Sergio Bernales García de Cañete”. Donde llega a los resultados fundamentales que el déficit semántica está relacionado con las palabras, expresiones y el déficit sintaxis se altera la estructura gramatical. Por otro lado, manifestaron que en los trabajados académicos no hay un déficit semántico. Finalmente, menciona que las dificultades lingüísticas inciden en la producción científica tecnológica escrita, producción literaria escrita, y en redacción administrativa.

En resumen, los resultados alcanzados del presente estudio nos expresan sobre el desarrollo del nivel de comunicación escrita, como medio de la comunicación es la interacción para los estudiantes, el cual conlleva a la mejora de la redacción en los diferentes tipos de textos y discursos comunicativos.

\section{CONCLUSIONES}

Los resultados evidencian que, si existe una relación significativa entre el nivel de comunicación escrita, las deficiencias lingüísticas, y el logro de competencias en estudiantes ingresantes en una Universidad Pública del Cusco. Se encuentra un factor de correlación de Rho de Spearman $r=0,638^{* *}$, denotando la existencia de una correlación efectiva moderado entre el nivel de comunicación escrita y deficiencias lingüísticas. Por otro lado, el factor de correlación de Rho de Spearman $r=0,726^{* *}$ lo que indica una correlación efectiva moderado entre el nivel de comunicación escrita y el logro de competencias en estudiantes ingresantes en una Universidad Pública del Cusco. Finalmente, se presenta el factor de correlación de Rho de Spearman $r=0,651 * *$ indica la existencia de una correlación positiva moderado entre las deficiencias lingüísticas y logro de competencias en estudiantes ingresantes en una Universidad Pública del Cusco. Por consiguiente, mientras exista un nivel alto de nivel de comunicación escrita y deficiencias lingüísticas habrá un buen nivel de logro de competencias en estudiantes ingresantes en la UNSAAC.

El nivel de comunicación en estudiantes ingresantes en una Universidad Pública del Cusco, se ha determinado que un 45,07\% muestra que tiene un nivel de comunicación escrita un nivel "bajo" en redacción de administrativa. De igual forma, el 43,66\% de los encuestados tienen un nivel de comunicación escrita "bajo" en redacción científica tecnológica. Así mismo, un 42,96\% expresaron que un nivel de comunicación escrita es "bajo" en redacción literaria. 
El nivel de las deficiencias lingüísticas en estudiantes ingresantes en una Universidad Pública del Cusco, se ha determinado en un 45,77\% de los encuestados expresaron que las deficiencias lingüísticas en un nivel “alto" en cuanto déficit semántico. De igual forma, se observa que un $40,85 \%$ de los encuestados indicaron que las deficiencias lingüísticas en nivel "alto" en cuanto déficit sintáctico. Así mismo, muestra un 39,44\% de los encuestados manifestaron que las deficiencias lingüísticas en un nivel "alto" en cuanto déficit ortográfico. Por otro lado, las deficiencias lingüísticas en un nivel "alto" en cuanto a déficit morfológico con 36,62\%.

La variable logro de competencias podemos indicar que el $47,89 \%$ de estudiantes ingresantes en una Universidad Pública del Cusco, tienen un nivel "regular", un 30,99\% con nivel de logro de competencia "bueno" y un $12.68 \%$ un nivel deficiente, y tan solo $8,45 \%$ un nivel de "muy bueno" en el logro de competencias en estudiantes ingresantes en la Universidad Nacional de San Antonio Abad del Cusco.

\section{LISTA DE REFERENCIAS}

Alonso-Cortés Manteca, Á. (2002). Lingüística. Cátedra Madrid Excelente. https://dialnet.unirioja.es/servlet/libro?codigo $=168340$

Arciniegas Lagos, E., \& López Jiménez, G. S. (2012). La escritura en el aula universitaria: Estrategias para su regulación. Programa Editorial UNIVALLE.

Bardales Balarezo, J. F. (2017). Deficiencias jurídicas en la aplicación del régimen de contratación administrativa de servicios (CAS) [Tesis, Universidad Nacional Hermilio Valdizan Huánuco]. http://repositorio.unheval.edu.pe/handle/UNHEVAL/2786

Beneitone, P., Esquetini, C., González, J., Maletá, M. M., Siufi, G., \& Wagenaar, R. (2007). Reflexiones y Perspectivas de la Educación Superior en América Latina Informe Final-Proyecto Tuning-América Latina 2004-2007. Universidad de Deusto.

Bravo Salinas, N. H. (2007). Competencias Proyecto Tuning-Europa, Tuning. - América Latina.

http://acreditacion.unillanos.edu.co/contenidos/cpacitacion_docente_2semestre_ 2007/competencias_proyectotuning.pdf

Cortés Moreno, M. (2000). Guía para el profesor de idiomas: Didáctica del español y segundas lenguas. Octaedro. 
Cronbach, L. J. (1951). Coefficient alpha and the internal structure of tests. Psychometrika, 16(3), 297-334.

Dale, M. (1992). Habilidades gerenciales. Legis Editores.

Estévez Fuertes, N., Gómez, J. R., \& Carbonell, M. (2011). El reto de la comunicación escrita en el siglo XXI. Quaderns de filología. Estudis lingüístics, XVI(24), 9-19.

Fonseca Yerena, M. del S., Correa Pérez, A., Pineda Ramírez, M. I., \& Lemus Hernández, F. J. (2011). Comunicación oral y escrita (1. ${ }^{\mathrm{a}}$ ed.). Prentice Hall. https://www.academia.edu/22962063/Comunicaci\%C3\%B3n_oral_y_escrita_1e d_-_Socorro_Fonseca_Alicia_Correa_y_Otros

García Cabrero, B., Loredo Enríquez, J., Luna Serrano, E., \& Rueda Beltrán, M. (2008). Modelo de Evaluación de Competencias Docentes para la Educación Media y Superior. Revista Iberoamericana de Evaluación Educativa, 1(3e), 97-108.

Gavidia Anticona, J. A. (2018). Deficiencias Lingüísticas en Relación con la Comunicación Escrita de los Estudiantes de Derecho y Ciencias Políticas-UNH - 2016 [Tesis, Universidad Nacional de Huancavelica]. http://repositorio.unh.edu.pe/handle/UNH/1441

Godoy Lenz, P., \& Zilliani Illanes, M. E. (2007). Guía: NEE en el Nivel de Educación Parvularia. Necesidades educativas especiales asociadas a lenguajes $y$ aprendizaje. Educación Especial. https://especial.mineduc.cl/recursos-apoyo-alaprendizaje/recursos-las-los-docentes/guia-nee-nivel-educacion-parvularia/

Gutiérrez Castillo, V. A., López Heredia, A., Salazar González, C., \& Ibarra Ramírez, L. G. (2012). Comunicación oral y escrita I (2. ${ }^{\mathrm{a}}$ ed.). Servicios Editoriales Once Ríos. https://www.freelibros.me/libros/comunicacion-oral-y-escrita-i-vicente-agutierrez-castillo

Hartle, F. (1995). How to Re-engineer Your Performance Management Process. Kogan Page.

Martínez Martínez, I. (2002). Un estudio contrastivo español-japonés. Fundación Canon - Carabela, 51, 47-67.

Martínez Solís, M. C. (2002). Estrategias de lectura y escritura de textos: Perspectivas teóricas y talleres. Universidad del Valle, Escuela de Ciencias del Lenguaje.

Mounin, G., \& Ter-Sakarian, G. (1979). Lingüística y filosofía. Gredos. https://dialnet.unirioja.es/servlet/libro?codigo=103969 
Pinales Rodríguez, D. G., \& Lagunas Beltrán, I. N. (1998). Comunicación oral y escrita. Trillas.

Rojas Saldaña, N. H. (2013). Deficiencias lingüísticas en relación a la comunicación escrita en estudiantes del ISTP «Sergio Bernales García» de Cañete [Tesis, Universidad de San Martín de Porres]. http://www.repositorioacademico.usmp.edu.pe/handle/usmp/1145

Snyder, A. V., \& Ebeling, H. W. (1992). Targeting a Company's Real Core Competencies. Journal of Business Strategy, 13(6), 26-32. https://doi.org/10.1108/eb039524

Toro Jaramillo, I. D., \& Parra Ramírez, R. D. (2006). Método y conocimiento: Metodología de la investigación: investigación cualitativa/investigación cuantitativa (1. ${ }^{\mathrm{a}}$ ed.). Universidad Eafit. 